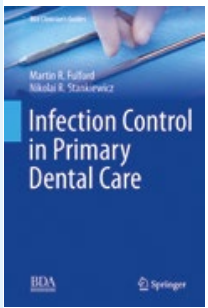

\section{BDJ CLINICIAN'S GUIDES: INFECTION CONTROL IN PRIMARY DENTAL CARE}

Martin R. Fulford and Nikolai R. Stankiewicz; 2019; Springer; £109.99 (£85.24 for BDA members when bought from the BDA shop); pp. 130; ISBN: 978-3-030-16306-8

Infection control in primary dental care ${ }^{1}$ is a concise, informative overview of infection control relevant to a dental setting. This clinical guide draws in your attention initially by describing the evolution and history of infection control. The different historical theories previously believed responsible for the spread of infection and high mortality rates associated with surgery are summarised by the authors. The constant struggle of the progressive clinicians to change the common beliefs is evident. Some barriers to infection control such as cost and time are clearly still issues to this day. The important topic of reducing our impact on the environment is touched on, with emphasis on finding a balance between single use items verses the ability to decontaminate or sterilise instruments.

The guide gives further background information of the dynamic relationship between microbes and humans, giving the reader further insight into the crucial need for an effective, reliable decontamination protocol in practice. These chapters on background science certainly increase the reader's understanding and reasoning behind vaccination of dental care professionals and the decontamination regimes described later in the handbook.

Further chapters go on to describe the different equipment available for decontaminating and sterilising instruments. As the book is not specific to one country, no guidelines or minimum standards are explicitly set out, more an overview to educate and inform the dental team, enabling clinicians to make a better informed decision with regard to infection control in practice.

Both authors are experienced in microbiology and in dental practice, making the information provided highly applicable to practice. The intended audience for this guide includes all dental care professionals, and the clinical guide caters to this broad group very well, with applications ranging from a dentist setting up or renovating a practice, to the dental nursing staff working daily in the decontamination unit.

Overall, this clinical guide to infection control is informative for the dental care professional. Interest is generated through the well written historical overview of the spread of infection, and the guide educates the reader in the background science, allowing them to make more informed decisions going forward. Infection control is a dynamic issue further progressing with new science and technology, and a sound understanding is essential for all clinical personnel.

By Miriam Crowley

\footnotetext{
Reference

1. Fulford M R, Stankiewicz N R. Infection Control in Primary Dental Care. Springer: Heidelberg, 2019. Available at https://shop.bda.org/icpdc (accessed December 2019).
}

\section{NE water fluoridation gains momentum}

Support for community water fluoridation continues to gain momentum with the unveiling of a new web page supporting schemes in the North East of England (https://onepartpermillion. co.uk/north-east). The web page is part of the One Part Per Million campaign initiated and funded by the local dental committee movement and is in readiness for public consultations in 2020 .

Local authorities in Northumberland and Durham have submitted formal letters to the Secretary of State for Health and Social Care, Matt Hancock, proposing the two new community water fluoridation schemes. The Durham scheme will cover Sunderland and South Tyneside.

At the heart of the website is research published in 2019 and carried out locally by members of the public health team which shows that nearly $40 \%$ of children living in deprived areas with non-fluoridated water have teeth which are decayed, missing or filled (dmft). This contrasts starkly with fluoridated areas, as well as more affluent areas, where the level is down to less than $10 \%$.

The process that a local authority must undertake to achieve water fluoridation is lengthy and complex. A letter to the Secretary of State must be followed by a public consultation with the opportunity for local people to ask questions and express their views. Finally, a request is made to the water company to add enough fluoride to reach the optimum one part per million ratio.

\section{Sheffield practice offers free mouth cancer exams}

To support November's Mouth Cancer Action Month, the one80 Dental Centre for Advanced Dentistry in Sheffield offered free oral health checks with mouth cancer examinations throughout November. Pictured is Nigel Rosenbaum, Principal and Owner.

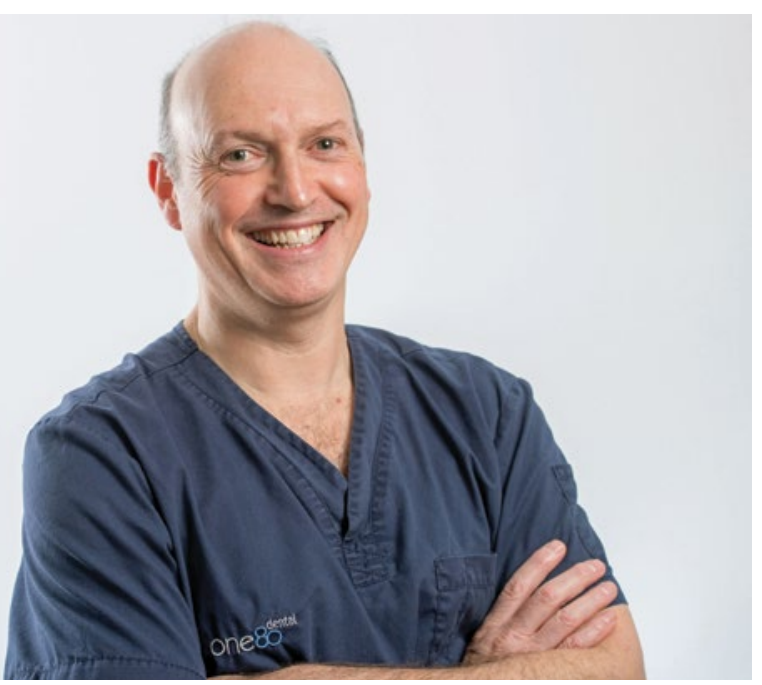

